\title{
Tromboembolismo pulmonar masivo con respuesta exitosa a trombolisis farmacológica. Caso clínico
}

\author{
JOSÉ LUIS LEAL ${ }^{a}$, PILAR LORA, \\ GIOVANNI ENCISO, ENRIQUE REYNOLDS
}

Facultad de Medicina Sede

Oriente, Universidad de Chile.

Unidad de Cuidados Intensivos,

Hospital Santiago Oriente.

Santiago de Chile.

${ }^{a}$ Becario de Medicina Interna,

Sede Oriente, Universidad

de Chile.

Recibido el 10 de agosto de 2009, aceptado el 23 de junio de 2010.

Correspondencia a: José Luis Leal Fernández. Tabancura 1623 Departamento 1901, Vitacura, Santiago de Chile. Fax: 2170440 E-mail: jlleal@gmail.com

\section{Massive pulmonary thromboembolism treated successfully with streptokinase. Report of one case}

\begin{abstract}
Massive pulmonary thromboembolism has a high mortality. Early thrombolysis is the treatment of choice. We report a 79-year-old man admitted in shock. A chest angio-CAT scan showed a massive pulmonary thromboembolism. A transthoracic echocardiography showed a right cardiac dysfunction. Although the patient was in hemodynamic instability, he was subjected to thrombolysis with streptokinase, assisted with noradrenaline support and invasive mechanical ventilation. Parenteral anticoagulation was started thereafter. A second echocardiography, performed 72 hours later showed an improvement in right ventricular function. The patient had a nosocomial pneumonia that was treated. Noradrenalin and mechanical ventilation were discontinued nine and 15 days after thrombolysis. A new angio-CAT scan, 23 days after the procedure, was normal. The patient was discharged in good conditions 27 days after admission.
\end{abstract}

(Rev Med Chile 2010; 138: 856-861).

Key words: Streptokinase: Thromboembolism; Tomography scanners, $x$-ray computed.
$\mathrm{E}$ 1 tromboembolismo pulmonar (TEP) masivo es una entidad clínica de altísima mortalidad. La sospecha clínica es fundamental para el tratamiento inmediato, que de no realizarse llevará a la muerte del paciente.

Presentamos un caso en que el diagnóstico se sospechó y buscó dirigidamente, lo que permitió realizar terapia de primera línea precozmente, obteniéndose excelente resultado.

\section{Caso clínico}

Hombre de 79 años, hipertenso, sin tratamiento farmacológico, autovalente.

Consultó en centro de atención primaria por cuadro brusco de epigastralgia y disnea intensa.
Se objetivó hipotensión $(70 / 30 \mathrm{mmHg})$, pulso irregular hasta $140 / \mathrm{min}$, mala perfusión distal, por lo que se inició volumen y dopamina en bomba de infusión continua (BIC). En nuestro centro se confirmó estado de shock pese a terapia descrita, sin ingurgitación yugular, ausencia de soplos o tercer ruido, sin signos de trombosis venosa. Electrocardiograma compatible con fibrilación auricular y signos de sobrecarga derecha (S1Q3T3) (Figura 1). Ecocardiografía transtorácica (ECTT) con signos de sobrecarga derecha. Angiografía por tomografía axial computada (angioTAC) de tórax demostró TEP masivo bilateral (Figura 2). En exámenes (Tabla 1) destacó acidosis metabólica, insuficiencia respiratoria, falla renal aguda y alteración de pruebas hepáticas. Ingresó a la Unidad de Cuidados 
Tabla 1. Exámenes del paciente a su ingreso

\begin{tabular}{|c|c|c|c|c|c|}
\hline $\mathrm{PAFi} / \mathrm{PCO}_{2}$ & $\begin{array}{c}\mathrm{HCO} 3 \\
(\mathrm{mEq} / \mathrm{L}) / \mathrm{pH}\end{array}$ & $\begin{array}{l}\mathrm{Na} / \mathrm{K} / \mathrm{Cl} \\
(\mathrm{mEq} / \mathrm{L})\end{array}$ & $\begin{array}{l}\text { BUN/Creat } \\
\text { (mg/dL) }\end{array}$ & $\begin{array}{c}\text { HTO (\%)/Hb } \\
\text { (mg/dL) }\end{array}$ & $\begin{array}{l}\text { Leuco }(\mathrm{K} / \mathrm{uL}) / \\
\text { PLTs }\left(\mathrm{mm}^{3}\right)\end{array}$ \\
\hline $190 / 32,4$ & $11,1 / 7,16$ & $138 / 6,3 / 105$ & $29 / 1,47$ & $46 / 15$ & $11.300 / 400.000$ \\
\hline $\begin{array}{l}\text { Lactato } \\
(\mathrm{mg} / \mathrm{dL})\end{array}$ & $\begin{array}{l}\text { Bili T/D/I } \\
\text { (mg/dL) }\end{array}$ & $\begin{array}{l}\text { AST/ALT } \\
\text { (UI) }\end{array}$ & $\begin{array}{l}\text { GGT/LDH } \\
\text { (UI/L) }\end{array}$ & $\begin{array}{c}\text { FA } \\
\text { (UI/L) }\end{array}$ & $\begin{array}{c}\text { Amilasa/Lipasa } \\
\text { (UI/L) }\end{array}$ \\
\hline 38,6 & $2,69 / 1,48 / 1,21$ & $811 / 533$ & 134 / 809 & 140 & $30 / 16$ \\
\hline
\end{tabular}

Entre paréntesis: Unidades de medida. PAFi: Indice Presión arterial de oxígeno / Fracción inspirada de oxígeno. Leuco: Leucocitos totales. Bili T / D / I: Bilirrubinemia Total / Directa / Indirecta. PLTs: Plaquetas totales. FA: Fosfatasas alcalinas.

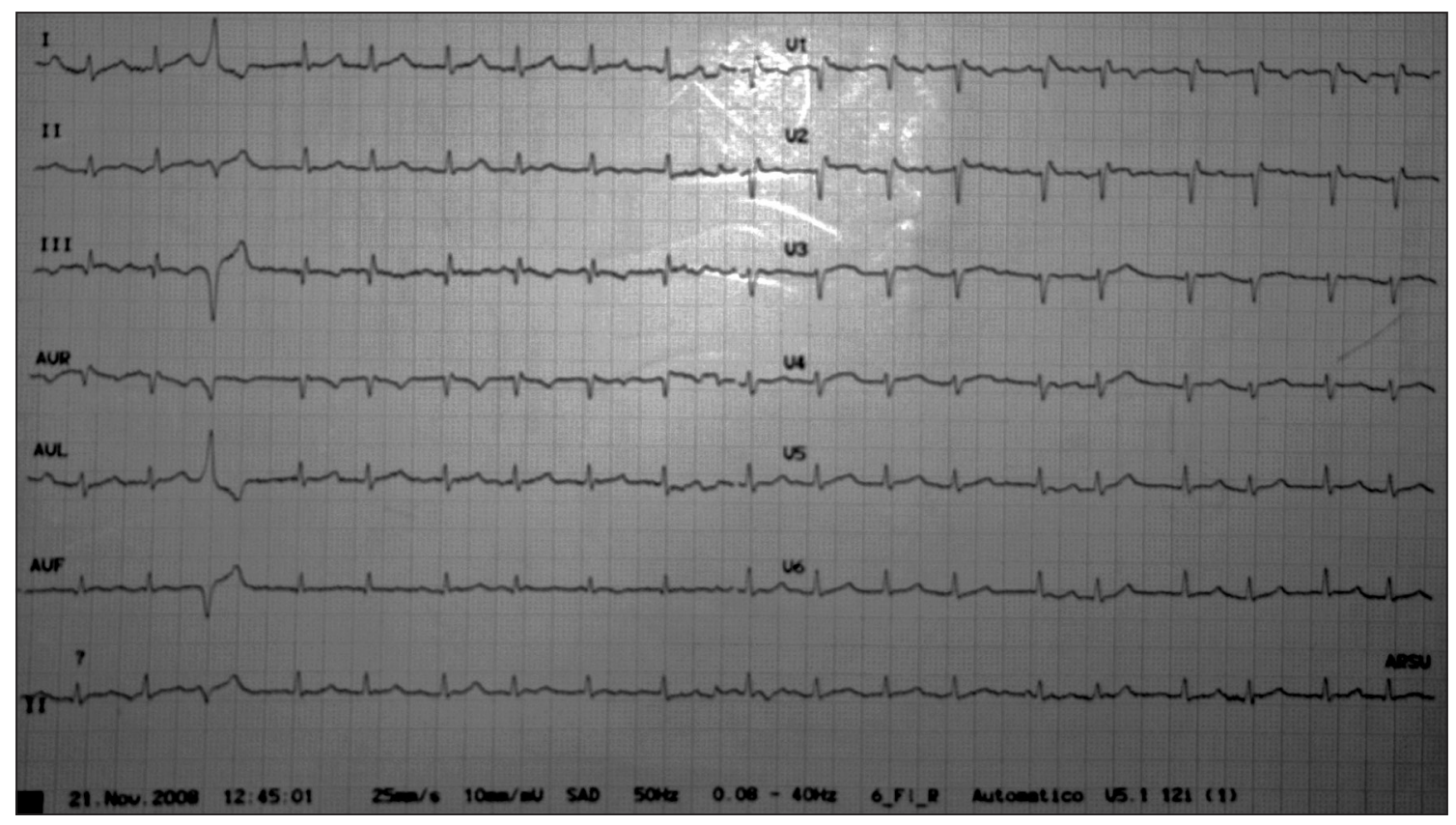

Figura 1. Electrocardiograma de ingreso en paciente con tromboembolismo pulmonar masivo. Se observa ritmo de fibrilación auricular, un extrasístole ventricular y S1Q3T3, esto último característico del TEP con sobrecarga ventricular derecha.

Intensivos (UCI), se inició noradrenalina (NA) y estreptokinasa (SK): $250.000 \mathrm{U}$ en 1 hora, seguido de 1.250.000 U en 23 horas. La presión cayó aun más, apareció bradicardia y compromiso de conciencia, por lo que se intubó y conectó a ventilación mecánica (VM), al tiempo que se suspendió transitoriamente la trombolisis. Ya estabilizado, se reinició infusión de SK, que completó por 24 horas, y se inició a continuación heparina en dosis anticoagulante por BIC. Evolucionó con requerimiento de altas dosis de NA en pos de mejoría de la perfusión distal y recuperación de la función renal; se cambió heparina por enoxaparina a las
48 horas de ingreso. Paulatinamente disminuyeron requerimientos de NA y de oxígeno. ECTT a las 72 horas de la trombolisis mostró mejoría de la motilidad del ventrículo derecho y buena función sistólica izquierda.

Posteriormente desarrolló neumonía intrahospitalaria (NIH), posiblemente aspirativa, ya que coincidió con regurgitación de papilla aportada por sonda nasogástrica. Se trató con imipenem y nutrición parenteral transitoriamente. Se aisló Acinetobacter baumannii y Staphylococcus aureus multisensible en vía aérea, completándose terapia antimicrobiana. 
Tromboembolismo pulmonar masivo con respuesta exitosa a trombolisis farmacológica. Caso clínico - J. L. Leal et al
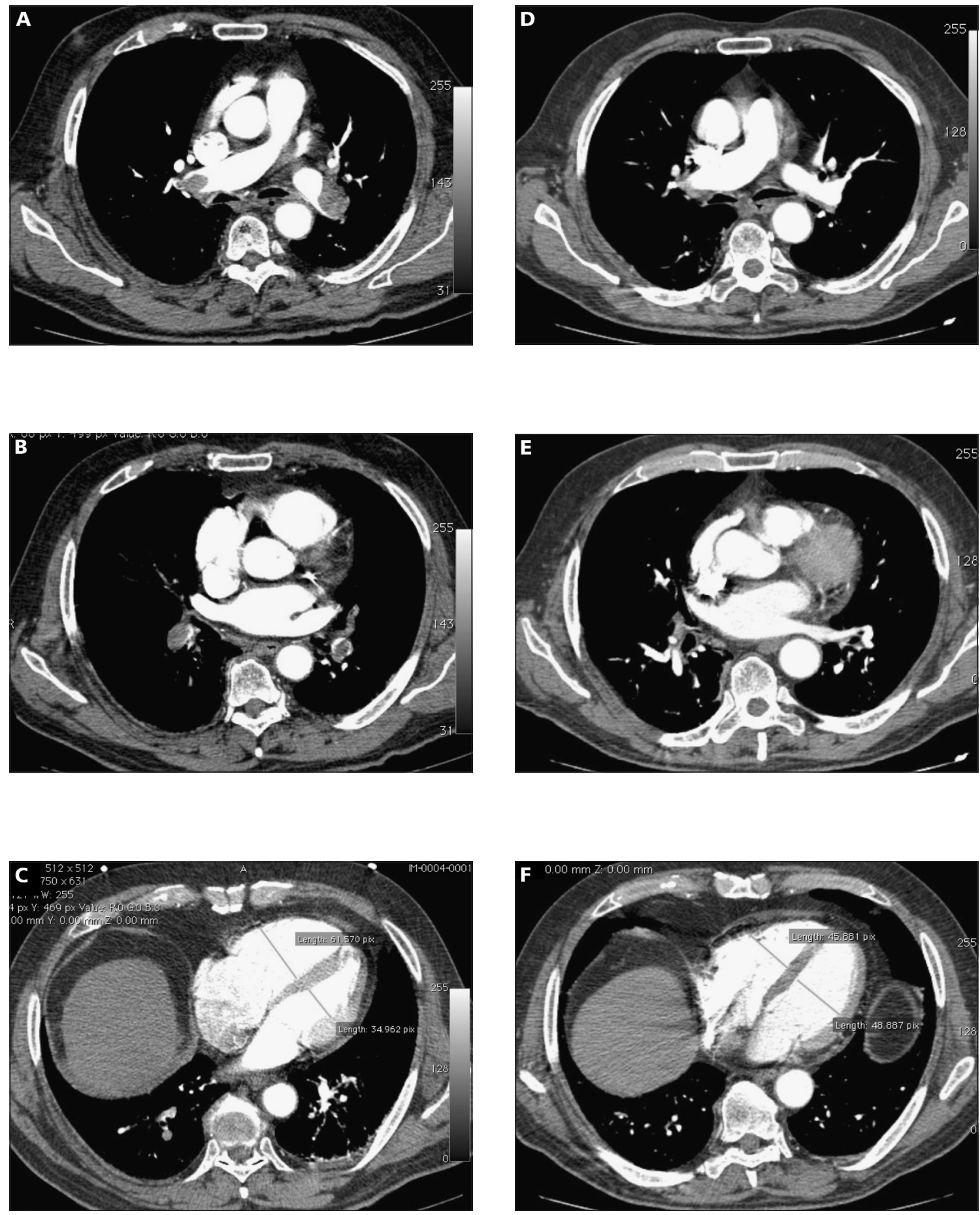

Figura 2. AngioTAC pulmonar de ingreso y de control. A la izquierda, se muestran imágenes de ingreso, con trombos centrales en ambas arterias pulmonares y que se continúan hacia ramas segmentarias y subsegmentarias. En 2.b. se puede observar además el "signo del riel". En 2 c. se observa dilatación del ventrículo derecho y desviación del septum interventricular a izquierda. A derecha, imágenes correlativas del AngioTAC de control a los 23 días de la trombolisis. No hay evidencias de focos hipodensos sugerentes de trombos. En 2 f. se observa normalización del tamaño del ventrículo derecho y del tabique interventricular. 
Se suspendió NA al noveno día de ingreso. Los requerimientos de oxígeno fueron lentamente en descenso. El destete, dificultado por agitación y desadaptación, requiriendo distintos esquemas de sedación, se completó el día 15. Se realizó traslape a tratamiento anticoagulante oral (TACO). A los 20 días se trasladó a intermedio. AngioTAC torácica al día 23 sin evidencias de trombos en arteria pulmonar ni sobrecarga de corazón derecho.

De alta al día 27 de hospitalización. A 6 meses del cuadro el paciente retomó sus actividades de la vida diaria y permanece en TACO.

\section{Discusión}

La presentación clínica de nuestro paciente es característica del TEP masivo o de alto riesgo ${ }^{1}$. Corresponde a 5\% de los casos, y conlleva un alto riesgo de mortalidad, que globalmente varía entre 24,5 y $68 \%$ de acuerdo a la forma de presentación ${ }^{2}$. El pronóstico no ha variado con la mejoría de los métodos diagnósticos y terapéuticos ${ }^{1,2}$.

La fisiopatología del TEP masivo es atribuible a falla del ventrículo derecho contextuada en una obstrucción completa del flujo pulmonar, con hipertensión pulmonar secundaria, llevando a compromiso de la precarga del ventrículo izquierdo y falla cardíaca global ${ }^{3}$. El grado de compromiso de la circulación pulmonar dependerá además del volumen de los émbolos y de la función pulmonar de base ${ }^{3}$.

El abordaje es en paralelo con un plan diagnóstico y otro de soporte. Debe iniciarse reposición de volumen con solución salina y tratamiento vasopresor. Existe poca evidencia avalando el uso de terapia inotrópica ${ }^{4,5}$. Según necesidad, oxigenoterapia, intubación y $\mathrm{VMI}^{5}$.

El diagnóstico conjuga la sospecha clínica con exámenes dirigidos. El algoritmo diagnóstico está bien descrito ${ }^{5}$. En cuanto a imágenes, el examen de elección es el AngioTAC helicoidal torácico, con sensibilidad cercana a $83 \%$ que aumenta a $90 \%$ combinado con venografía de extremidades inferiores ${ }^{6}$, factible de realizarse en el mismo momento. Asimismo, otorga información pronóstica al medir tamaño del ventrículo derecho, y permite descartar diagnósticos alternativos ${ }^{7}$. El algoritmo prosigue con la ECTT, que evalúa función ventricular derecha, estratifica el riesgo inicial (alto, intermedio o bajo) con la ventaja de ser bedside, y ofrece la potencialidad de guiar el tratamiento, sea al inicio o en el seguimiento ${ }^{8}$. Ambos exámenes se realizaron al ingreso y en el seguimiento de nuestro caso.

Como tratamiento del TEP con compromiso hemodinámico, se describen: trombolisis farmacológica sistémica o intratrombo, fragmentación mecánica, trombectomía mecánica y embolectomía quirúrgica.

La trombolisis es la terapia de primera línea en TEP de alto riesgo (shock cardiogénico o hipotensión arterial persistente) con pocas contraindicaciones absolutas. Varios estudios han demostrado rápida resolución y gran beneficio en parámetros hemodinámicos. El estudio de Tibbutt et al, mostró a las 72 horas de tratamiento con SK, $80 \%$ de mejoría en el índice cardíaco y $40 \%$ de baja en la presión de arteria pulmonar'. Estudios con urokinasa y alteplase ${ }^{8,10}$ muestran resultados similares. No hay diferencias entre urokinasa y $\mathrm{SK}^{11}$. El activador recombinante de plasminógeno tisular (rtPA) sería levemente más rápido en resolver las anomalías hemodinámicas comparado con urokinasa y $\mathrm{SK}^{12}$. Infusiones directas en arteria pulmonar no ofrecen ventajas con respecto a trombolisis sistémica ${ }^{13}$. Las dosis recomendadas se muestran en la Tabla 2.

Hasta 92\% de pacientes son "respondedores", basados en mejoría clínica y ecocardiográfica a las 36 horas $^{14}$. El mayor beneficio de la trombolisis se observa en las primeras 48 horas, pero se ha demostrado utilidad hasta 14 días después de iniciados los síntomas ${ }^{15}$.

\section{Tabla 2. Dosis de trombolíticos recomendadas para el tratamiento del tromboembolismo pulmonar masivo (adaptada de referencia 16)}

\begin{tabular}{|c|c|}
\hline \multirow[t]{2}{*}{ Estreptokinasa } & $\begin{array}{l}250.000 \mathrm{UI} \text { iv en } 30 \text { minutos, luego } \\
100.000 \mathrm{UI} \text { / hora en } 12 \text { a } 24 \text { horas }\end{array}$ \\
\hline & $\begin{array}{l}\text { Régimen acelerado: } 1.500 .000 \text { iv en } \\
2 \text { horas }\end{array}$ \\
\hline \multirow[t]{2}{*}{ Urokinasa } & $\begin{array}{l}4.400 \mathrm{UI} / \mathrm{Kg} \text { en } 10 \text { minutos, luego } \\
4.400 \mathrm{UI} / \mathrm{Kg} / \mathrm{h} \text {. en } 12 \text { a } 24 \text { horas }\end{array}$ \\
\hline & $\begin{array}{l}\text { Régimen acelerado: } 3.000 .000 \text { en } \\
2 \text { horas }\end{array}$ \\
\hline rtPA & $\begin{array}{l}100 \mathrm{mg} \text { en } 2 \text { horas o } 0,6 \mathrm{mg} / \mathrm{kg} \text { en } 15 \\
\text { minutos (máximo } 50 \mathrm{mg} \text { ) }\end{array}$ \\
\hline
\end{tabular}

rtPA: activador recombinante de plasminógeno tisular. 
Se describe $13 \%$ de eventos hemorrágicos mayores y $1,8 \%$ de hemorragias intracraneales/fatales. Esta probabilidad disminuye cuando se usan métodos no invasivos para confirmar el TEP, siendo esta la conducta más ampliamente recomendada ${ }^{16}$. La trombolisis no está indicada en casos que no son de alto riesgo, pero podría ser considerada en TEP con riesgo intermedio (evidencia ecográfica de sobrecarga del ventrículo derecho o elevación de marcadores de injuria miocárdica), lo que actualmente es tema de discordia.

Si hay contraindicación absoluta o inefectividad de la trombolisis, se debe intentar fragmentación mecánica del trombo o embolectomía quirúrgica.

En cuanto a las terapias percutáneas, aunque la evidencia se limita a reportes o series de casos, se recomiendan como alternativa a la trombolisis cuando hay contraindicaciones absolutas para ella, como adyuvante cuando la trombolisis falla o como alternativa a la embolectomía quirúrgica ${ }^{16}$.

Para lograr la desobstrucción de las arterias pulmonares centrales, están descritas la fragmentación y aspiración de trombos y la embolectomía $^{17,18}$. Esto reduce significativamente la hipertensión pulmonar, la sobrecarga ventricular derecha y aumenta la superficie de contacto con el trombolítico. En una serie se evidenció una mejoría en 90\% de los pacientes con TEP masivo tratados con trombectomía mecánica percutánea ${ }^{17}$.

La trombectomía quirúrgica se describió en $1924^{19}$. Tradicionalmente ha tenido un rol de rescate en pacientes que necesitaron resucitación cardiopulmonar, con contraindicación de trombolisis o foramen oval permeable con trombo intracardíaco ${ }^{20}$. Actualmente se recomienda sólo en centros con alta experiencia y, con las técnicas quirúrgicas actuales, sería una herramienta útil en casos donde la trombolisis fracasó o está contraindicada ${ }^{16}$.

La anticoagulación es de regla ${ }^{1,5,16}$. Debe iniciarse inmediatamente, dado que disminuye la mortalidad y la recurrencia de eventos, incluso cuando no se ha confirmado el diagnóstico pero la sospecha es alta. La anticoagulación en caso de trombolisis con infusión de reteplase o alteplase se puede iniciar de inmediato; con SK o urokinasa debe utilizarse posterior a terminada la infusión. Lo recomendado es mantener por al menos cinco días y después pasar a TACO; se ha comunicado hasta tres veces más recurrencias en pacientes que iniciaron inmediatamente TACO comparados con inicio de heparina no fraccionada y que luego pasaron a $\mathrm{TACO}^{21}$. Se prefieren heparinas de bajo peso molecular (HBPM) o fondaparinux por menor riesgo de sangrado y ausencia de necesidad de monitorización, salvo en falla renal (aclaramiento de creatinina menor a $30 \mathrm{ml} / \mathrm{min}$ ) y en TEP de alto riesgo (no hay estudios en shock o hipotensión) donde se recomienda heparina no fraccionada ${ }^{18}$. Actualmente en evaluación, los inhibidores orales del factor Xa y IIa potencialmente podrían sustituir la anticoagulación parenteral inicial.

En resumen, la recomendación actual en casos de alta sospecha clínica es realizar un angioTAC de tórax, como en nuestro caso; confirmado el diagnóstico, si el paciente ingresa en shock (alto riesgo), trombolisis salvo contraindicación absoluta. Queremos destacar que esta terapia sigue siendo preferida en primera línea, y su efectividad queda claramente graficada en la evolución presentada en este caso. Si no hay shock, ECCT para evaluar compromiso de cavidades derechas y estratificar el riesgo. Si no hay compromiso ventricular derecho (bajo riesgo), entonces sólo anticoagulación; si se evidencia sobrecarga ventricular derecha (riesgo intermedio), anticoagulación y evaluar según cada caso el uso de trombolisis o terapias percutáneas intervencionales, lo que sigue siendo un tema controvertido, por lo cual hay estudios actualmente en curso.

\section{Referencias}

1. Konstantinides S. Acute pulmonary embolism. N Engl J Med 2008; 359: 2804-13.

2. Kucher N, Goldhaber S. Management of massive pulmonary embolism. Circulation 2005; 112: 28-32.

3. Wood K. Major pulmonary embolism: review of a pathophysiologic approach to the golden hour of hemodynamically significant pulmonary embolism. Chest 2002; 121: 877-905.

4. Layish D, Tapson V. Pharmacologic hemodynamic support in massive pulmonary embolism. Chest 1997; 111: 218-24.

5. Tapson VF. Acute pulmonary embolism. N Engl J Med 2008; 358: 1037-52.

6. Stein P, Fowler S, Goodman L, Gottschalk A, Hales C, Hull R, et al. Multidetector computed tomography for acute pulmonary embolism. N Engl J Med 2006; 354: 2317-27. 
7. Schoepf U, Kucher N, Kipfmueller F, Quiroz R, Costello P, Goldhaber S. Right ventricular enlargement on chest computed tomography: a predictor of early death in acute pulmonary embolism. Circulation 2004; 110: 3276-80.

8. Goldhaber S, Haire W, Feldstein M, Miller M, Toltzis $\mathrm{R}$, Smith J, et al. Alteplase versus heparin in acute pulmonary embolism: randomized trial assessing rightventricular function and pulmonary perfusion. Lancet 1993; 341: 507-11.

9. Tibbutt D, Davies J, Anderson J, Fletcher E, Hamill J, Holt J, et al. Comparison by controlled clinical trial of streptokinase and heparin in treatment of life-threatening pulmonary embolism. BMJ 1974; 1: 343-7.

10. Meyer G, Sors H, Charbonier B, Kasper W, Bassand J, Kerr I, et al. Effects of intravenous urokinase versus alteplase on total pulmonary resistance in acute massive pulmonary embolism: a European multicenter doubleblind trial. The European Cooperative Study Group for Pulmonary Embolism. J Am Coll Cardiol 1992; 19: 239 45.

11. Urokinase-streptokinase embolism trial. Phase 2 results. A cooperative study. JAMA 1974; 229: 1606-13.

12. Dalla-Volta S, Palla A, Santolicandro A, Giuntini C, Pengo V, Visioli O, et al. Alteplase combined with heparin versus heparin in the treatment of acute pulmonary embolism. Plasminogen activator Italian multicenter study 2. J Am Coll Cardiol 1992; 20: 520-6.

13. Verstraete M, Millerg, Bounameaux H, Charbonier B, Colle JP, Lecorf G, et al. Intravenous and intrapulmonary recombinant tissue-type plasminogen activator in the treatment of acute massive pulmonary embolism. Circulation 1988; 77: 353-60.
14. Meneveau N, Seronde M, Blonde M, Legalery P, DidierPetit K, Briand F, et al. Management of unsuccessful thrombolysis in acute massive pulmonary embolism. Chest 2006; 129: 1043-50.

15. Daniels L, Parker J, Patel S, Grodstein F, Goldhaber S. Relation of duration of symptoms with response to thrombolytic therapy in pulmonary embolism. Am J Cardiol 1997; 80: 184-8.

16. Torbicki A, Perrier A, Konstantinides S, Agnelli G, Galie $\mathrm{N}$, Pryszczyk P, et al. Guidelines on the diagnosis and management of acute pulmonary embolism. The Task Force for the Diagnosis and Management of Acute Pulmonary Embolism of the European Society of Cardiology (ESC). Eur Heart J 2008; 29: 2276-15.

17. Fava M, Loyola S, Huete I. Massive Pulmonary Embolism Treatment with Hydrolyser Thrombectomy Catheter. JVIR 2000; 11: 1159-64.

18. Timsit JF, Reynaud P, Meyerg, Sors H. Pulmonary embolectomy by catheter device in massive pulmonary embolism. Chest 1991; 100: 655-8.

19. Kirschner M. Ein durch die Trendelenburgsche Operation geheilter Fall von Embolie der Arteria pulmonalis. Arch Klin Chir 1924; 312.

20. Leacche M, Unic D, Goldhaber S, Rawn JD, Aranki S, Couper GS, et al. Modern surgical treatment of massive pulmonary embolism: results in 47 consecutive patients after rapid diagnosis and aggressive surgical approach. J Thorac Cardiovasc Surg 2005; 129: 1018-23.

21. Brandjes D, Heijboer H, Buller H, De Rijk M, Jagt H, Ten Cate J. Acenocoumarol and heparin compared with acenocoumarol alone in the initial treatment of proximal-vein thrombosis. N Engl J Med 1992; 327: 1485-9. 